\title{
Performance evaluation of a motor-imagery- based EEG-Brain computer interface using a combined cue with heterogeneous training data in $\mathrm{BCl}$-Naive subjects
}

Donghag Choi ${ }^{\dagger}$, Yeonsoo Ryu ${ }^{\dagger}$, Youngbum Lee ${ }^{\dagger}$ and Myoungho Lee

* Correspondence: mhlee@yonsei. ac.kr

Department of Electrical and Electronic Engineering, Yonsei University, 134 Shinchon-dong, Seodaemun-gu, Seoul, 120-749, Korea

\begin{abstract}
Background: The subjects in EEG-Brain computer interface (BCl) system experience difficulties when attempting to obtain the consistent performance of the actual movement by motor imagery alone. It is necessary to find the optimal conditions and stimuli combinations that affect the performance factors of the EEG-BCl system to guarantee equipment safety and trust through the performance evaluation of using motor imagery characteristics that can be utilized in the EEG-BCI testing environment.
\end{abstract}

Methods: The experiment was carried out with 10 experienced subjects and 32 naive subjects on an EEG-BCl system. There were 3 experiments: The experienced homogeneous experiment, the naive homogeneous experiment and the naive heterogeneous experiment. Each experiment was compared in terms of the six audio-visual cue combinations and consisted of 50 trials. The EEG data was classified using the least square linear classifier in case of the naive subjects through the common spatial pattern filter. The accuracy was calculated using the training and test data set. The $p$-value of the accuracy was obtained through the statistical significance test.

Results: In the case in which a naive subject was trained by a heterogeneous combined cue and tested by a visual cue, the result was not only the highest accuracy $(p<0.05)$ but also stable performance in all experiments.

Conclusions: We propose the use of this measuring methodology of a heterogeneous combined cue for training data and a visual cue for test data by the typical EEG-BCl algorithm on the EEG-BCl system to achieve effectiveness in terms of consistence, stability, cost, time, and resources management without the need for a trial and error process.

Keywords: EEG, Brain Computer Interface, Motor Imagery, Heterogeneous and Homogeneous Combined Cue 


\section{Background}

Recently, BCI technology has progressed as state-of-the-art medical devices to control and communicate with applicable accessories such as artificial limbs, prosthetic and wheelchair using the signal of brain activity [1-3]. There have been numerous studies of brainwaves collected electrically from brain activity on the cortex related to the brain computer interface [4-6]. Other research has investigated the types of electrical brain activity that can be used to implement the EEG-BCI systems [7-9].

The electroencephalographic (EEG) mu rhythm is an $8-13 \mathrm{~Hz}$ rhythm generated by the sensorimotor cortex that is most prominent when subjects are resting and is attenuated or abolished when subjects move or observe biological movements $[10,11]$. The $\mu$-rhythm is capable of transforming by itself as opposed to the brain activity events [12]. Motor imagery implies a thought activity of imagining of physical movement. Without such physical activity it can transform the $\mu$-rhythm within the sensory motor cortex [13]. In other words, when imagining a hand movement or actually moving the hand, ERD (Event-Related Desynchronization) occurs around the $\mu$-rhythm area within the sensory motor cortex $[14,15]$.

However, the subjects in EEG-BCI system experience difficulties when attempting to obtain the consistent performance of the actual movement by motor imagery alone $[7,16]$. Hence, it is necessary to find the condition that affect the performance factors of the EEG-BCI system to guarantee equipment safety and trust through an evaluation of the performance of the EEG-BCI system using motor imagery characteristics that can be utilized in the EEG-BCI testing environment.

In a naive homogeneous training data experiment, Kim found that the types of training data do not affect the level of accuracy [16]. In an homogeneous training data experiment, Lee carried out a BCI experiment with experienced subjects and crosscompared three classifiers of combined cues (i.e., audio-visuals cues), analyzing the levels of statistical significance and cross-correlation [17]. However, Lee's study lacked statistical confidence, as the subjects in the experiment built experience based only on ten trials. Thus, in addition to the sample size limitation, the experiment also lacked any consideration of naive subjects.

In an effort to mitigate the sample size issue and include additional variables designed to improve the concentration of the subjects. Furthermore, while the existing combined cue experiment is based on homogeneous training data, this experiment is based on the heterogeneous data, which provides a useful comparative analysis. It should be noted that for the heterogeneous case, the cue speed is a mix of four- and two-second durations. Hence, the key comparisons made here are the naive homogeneous training data experiment and heterogeneous training data experiment.

\section{Methods}

\section{Subject and data acquisition}

The experiment was carried out with 10 experienced subjects aged $23.9 \pm 2.5$ and 32 naive subjects aged $23.5 \pm 1.8$ without encephalopathy, mental health disorder and selfmutilation. There were 3 experiments: The experienced homogeneous experiment, the naive homogeneous experiment and the naive heterogeneous experiment. The characteristics of each experiment are shown in Table 1. 
Table 1 Characteristics of the experiments

\begin{tabular}{|c|c|c|c|}
\hline $\begin{array}{c}\text { Characteristics of } \\
\text { experiment }\end{array}$ & $\begin{array}{l}\text { Experienced homogeneous } \\
\text { experiment }\end{array}$ & $\begin{array}{c}\text { Naive homogeneous } \\
\text { experiment }\end{array}$ & $\begin{array}{c}\text { Naive heterogeneous } \\
\text { experiment }\end{array}$ \\
\hline Experience of subject & Experienced & \multicolumn{2}{|c|}{ Naïve (i.e., no experience) } \\
\hline Number of subjects & 10 men/women & \multicolumn{2}{|c|}{32 men/women } \\
\hline $\begin{array}{l}\text { Average age of } \\
\text { subjects }\end{array}$ & $23.9 \pm 2.5$ & \multicolumn{2}{|c|}{$23.5 \pm 1.8$} \\
\hline $\begin{array}{l}\text { Stimuli type of } \\
\text { training data }\end{array}$ & Homogeneous & Homogeneous & Heterogeneous \\
\hline $\begin{array}{l}\text { Duration of a cue in } \\
\text { one trial }\end{array}$ & 7 seconds & 7 seconds & 6 or 8 seconds \\
\hline Number of trials & \multicolumn{3}{|c|}{50 trials } \\
\hline
\end{tabular}

The block diagram of the EEG-BCI system showed the signal acquisition and process in Figure 1. The brain activity signals were extracted at specific locations using a golden disk electrode with a hole. The locations of F3, Fz, F4, FC1, FCz, FC2, C3, C1, $\mathrm{Cz}, \mathrm{C} 2, \mathrm{C} 4, \mathrm{CP} 1, \mathrm{CP} 2, \mathrm{P} 3, \mathrm{Pz}$, and P4 were selected according to the extended international 10/20 system. The impedance of each electrode was $5 \mathrm{k} \Omega$ or less. The EEG acquisition and management system (model: WEG-32, Laxtha Inc. Korea) generated EEG data using an input signal from an amplifier and transferred the EEG data to a notebook computer connected by a USB interface.

The EEG data was classified using the least square (LS) linear classifier in case of the naive subjects through the common spatial pattern (CSP) filter. We calculated the accuracy using the training data set and test data set. The $p$-value of the accuracy was obtained through the statistical significance test (i.e., t-test). In case of the experienced subjects, we used three types of classifiers: the least square (LS) linear classifier using a linear matrix equation and a pseudo inverse matrix, the support vector machine (SVM) using a pattern classifier based on structural risk minimization, and linear

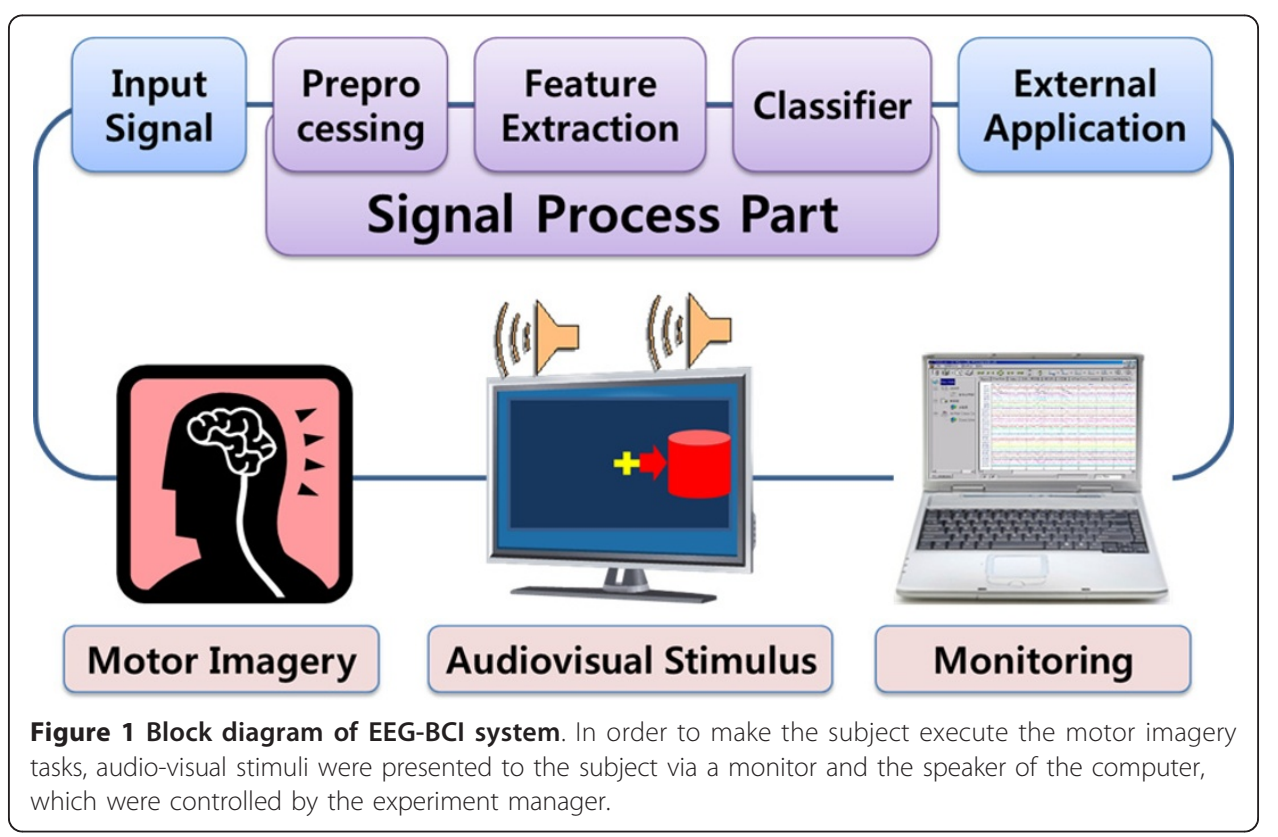


discriminant analysis (LDA) using the method reducing the dimension of the data feature vector by maximizing the ratio between the intra-classes-distribution and the inter-classes-distribution. The accuracy as to how the EEG-BCI reading of the brainwave collected matches the actual instructed data is then recorded.

\section{Experimental procedure}

There are six cue-combinations in total, comprised of two visual cues, two auditory cues and two combined cues as shown in Table 2.

Each combination consists of 50 trials. For the training data, the time for each cue is 6 or 8 seconds followed by 2 seconds of a blank screen, 2 seconds of a fixation cross and 2 or 4 seconds of cue intervals to train the motor imagery. For the test data, the time for each cue is 7 seconds, and this is followed by 2 seconds of a blank screen, 2 seconds of a fixation cross and 3 seconds of cue intervals to test the motor imagery. The subject imagines the motor imagery according to the audio-visual cue instructions presented on the computer monitor and speaker that are operated by the experiment manager.

Table 3 shows one trial of cue presentations using a cue. If the cue presented only visually, then the cue is referred to as a visual cue. If the cue presented only as an auditory cue, then the cue is referred to as an auditory cue, and if the cue presented as both an auditory and a visual cue, then the cue is considered as a combined cue. The cueing method and presenting duration are randomly distributed, eliminating the possibility of prediction through training.

\section{Experienced homogeneous experiment}

The experimental results of the experienced subjects using homogeneous training data derived from a homogeneous stimulus having the same cue time for motor imagery is presented for 3 seconds in case of the training data and 3 seconds in case of the test data, as shown in Table 3. For example, if we used the combined cue for the left motor imagery training tasks, a blank screen was presented to the experienced subject for 2 seconds and the fixation cross was continuously presented for 2 seconds. Finally, a red cylinder on the left side of the monitor with the beep sound and the "left" sound of the speaker were presented for 3 seconds simultaneously for motor imagery.

\section{Naive homogeneous experiment}

The experimental results of the naive subjects using the homogeneous training data derived from a homogeneous stimulus having the same cue time for motor imagery is presented for 3 seconds for training and 3 seconds for testing as shown in Table 3. For

Table 2 Cue-combinations in the experiment

\begin{tabular}{ccc}
\hline Cue-combinations & Training & Testing \\
\hline VA & Visual Cue & Auditory Cue \\
\hline$A V$ & Auditory Cue & Visual Cue \\
\hline CA & Combined Cue & Auditory Cue \\
\hline CV & Combined Cue & Visual Cue \\
\hline VC & Visual Cue & Combined Cue \\
\hline$A C$ & Auditory Cue & Combined Cue \\
\hline
\end{tabular}

There are six cue-combinations as $\mathrm{VA}, \mathrm{AV}, \mathrm{CA}, \mathrm{CV}, \mathrm{VC}$, and $\mathrm{AC}$. For example, in the case of the CV combination, the experiment used the combined cue for training and the visual cue for testing. 
Table 3 One trial of cue presentation

\begin{tabular}{|c|c|c|c|c|c|c|}
\hline Experi-mental Phase & $\begin{array}{l}\text { Display } \\
\text { Blank } \\
\text { Screen }\end{array}$ & Display Fixation Cross & $\begin{array}{c}\text { Beep } \\
\text { for Starting }\end{array}$ & $\begin{array}{c}\text { Sound } \\
\text { "Left" or "Right" }\end{array}$ & Display Red Cylinder on Left or Right & $\begin{array}{l}\text { Beep } \\
\text { for Stopping }\end{array}$ \\
\hline Homo-geneous cue & 2 seconds & 2 seconds & & & 3 seconds & \\
\hline Hetero-geneous cue & 2 seconds & 2 seconds & \multicolumn{4}{|c|}{2 seconds or 4 seconds, randomly } \\
\hline \multirow[t]{2}{*}{$\begin{array}{l}\text { Stimuli } \\
\| \\
\text { Screen } \\
\text { and/or } \\
\text { Sound }\end{array}$} & & + & $\begin{array}{l}\text { "Beep" } \\
\text { "Beep" }\end{array}$ & $\begin{array}{l}\square(\|)) \\
\text { "Left" }\end{array}$ & + & $\begin{array}{c}5(j)) \\
\text { "Beep" }\end{array}$ \\
\hline & & & $\begin{array}{l}\text { "Beep") } \\
\text { "Beep" }\end{array}$ & $\begin{array}{c}\text { (Al)) } \\
\text { "Right" }\end{array}$ & & $\begin{array}{l}\text { "Beep" } \\
\text { "Beep" }\end{array}$ \\
\hline
\end{tabular}

One experiment consisted of 50 trials. One trial required 7 and 6 or 8 seconds for presenting the cue to each subject for a motor imagery time of 3 and 2 or 4 seconds with audio-visual stimuli presented randomly and unpredictably. 
example, if we used the visual cue for the right motor imagery training tasks, a blank screen was presented to the experienced subject for 2 seconds and the fixation cross was continuously presented for 2 seconds. Finally, a red cylinder on the right side of the monitor with the beep sound was presented for 3 seconds for motor imagery.

\section{Naive heterogeneous experiment}

The experimental results using the naive heterogeneous training data derived from 2 types of heterogeneous stimuli having a different cue time was presented for 2 or 4 seconds in case of the training data as shown in Table 3. For example, if we used the combined cue for the right motor imagery training tasks, a blank screen was presented to the experienced subject for 2 seconds and the fixation cross was continuously presented for 2 seconds. Finally, a red cylinder on the right side of the monitor with the beep sound and the "right" sound of the speaker were presented for 2 or 4 seconds simultaneously for motor imagery. The duration of the last phase in this trial was randomized.

\section{Results}

\section{Experienced homogeneous experiment}

The experiment with experienced subjects using homogeneous training data sought to investigate the responses to a combination of visual and auditory (i.e., audio-visual) cues. Furthermore, a comparative evaluation was done based on the three classifiers (LS, SVM, and LDA), followed by a statistical analysis to investigate the significance and correlation between the six combinations used in the audio-visual cue experiment.

Subject number 1 had an accuracy score of 0.8 for all three classifiers (LS, SVM, and LDA). The second highest performers were numbers 4 and 8, who scored above 0.6. Subject number 3 had the lowest accuracy mark for all three classifiers (LS, SVM, and LDA).

The plots in Figure 2 show the accuracy of the three classifiers (LS, SVM, and LDA) for all six combinations. It was found that the $\mathrm{CV}$ and $\mathrm{VC}$ combinations have higher

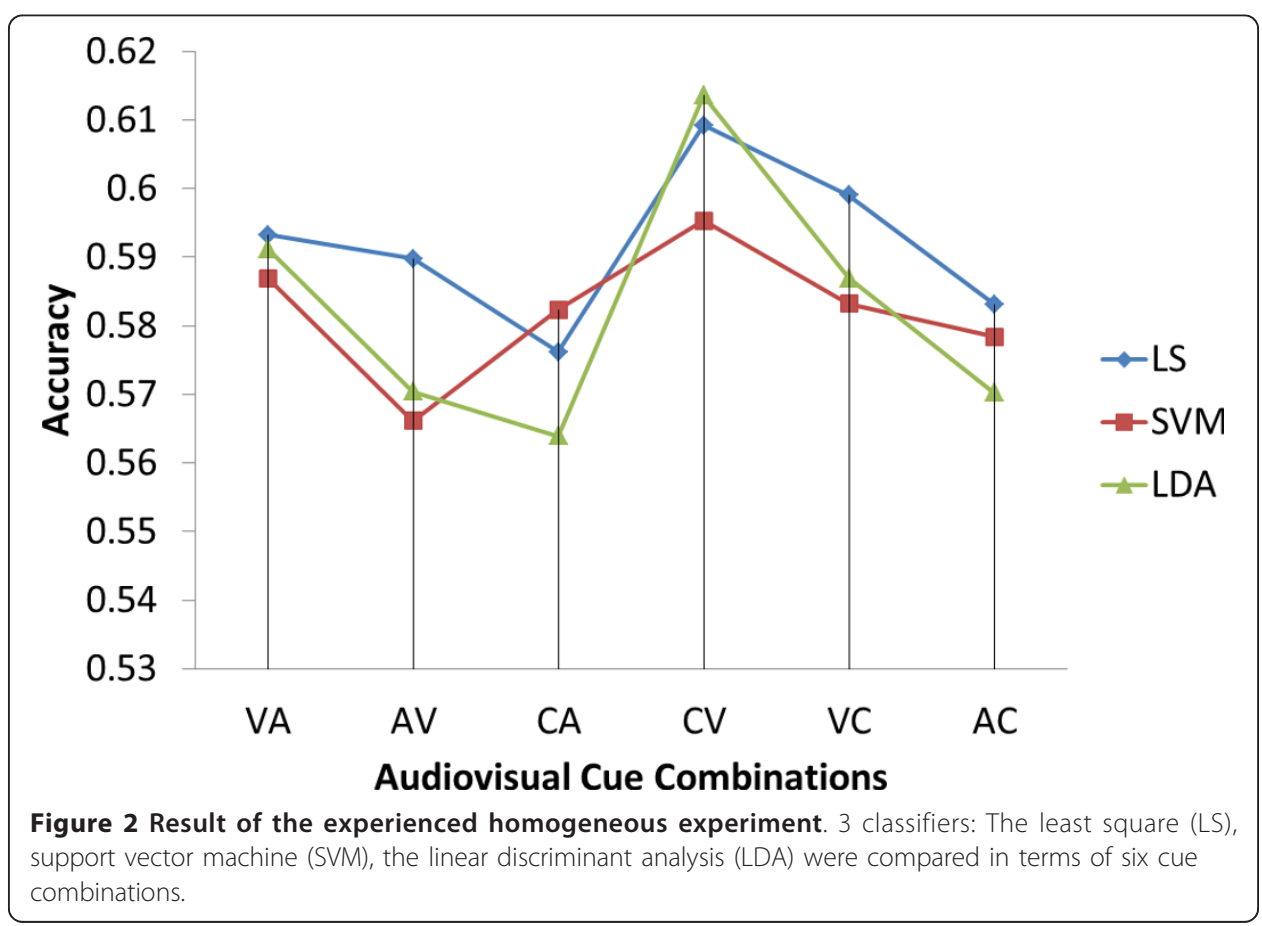




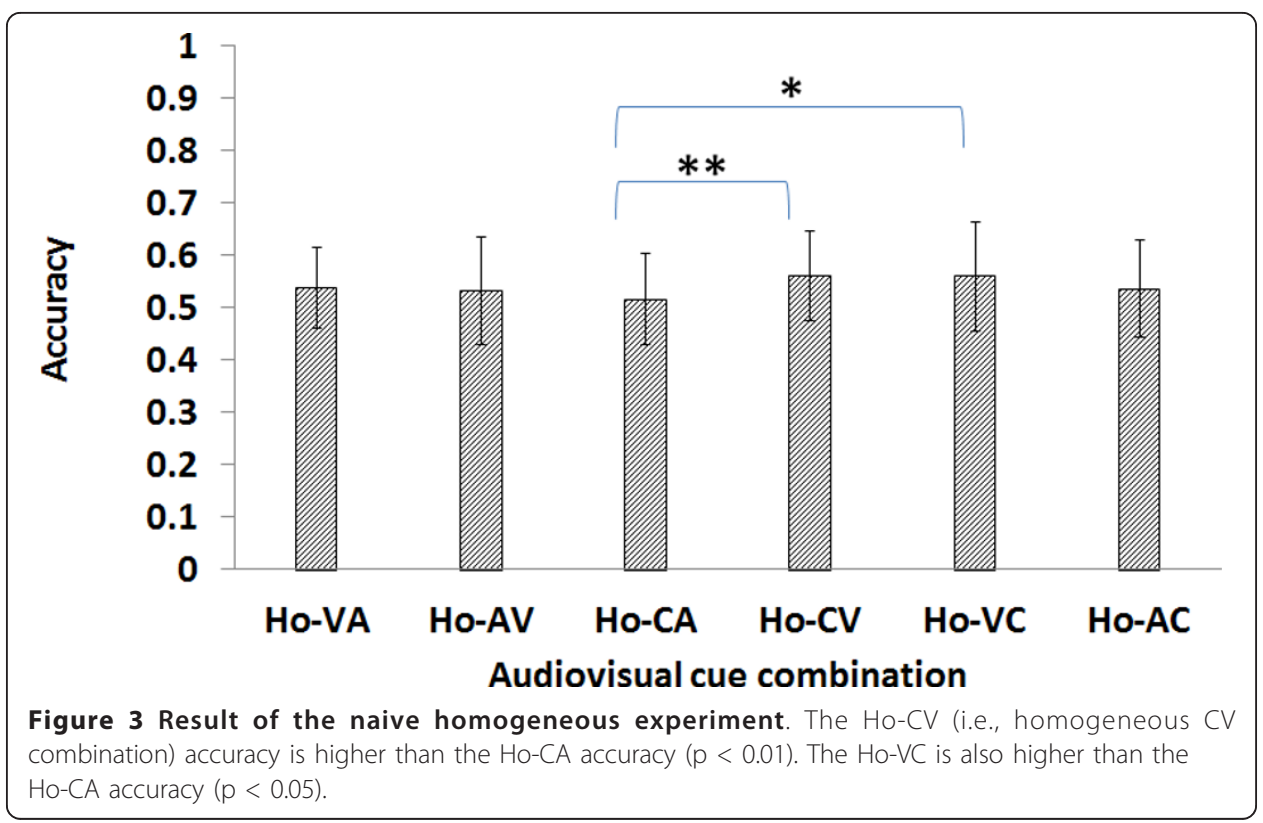

accuracy levels than the others for all three classifiers (LS, SVM, and LDA). The CV accuracy is higher than that of CA $(\mathrm{p}<0.05)$ in the case of LDA, while the other cases show no statistical significance.

\section{Naive homogeneous experiment}

Figure 3 shows the result of the experiment using homogeneous training data with the naive subjects in average accuracy of the six cue-combinations. Ho-CV shows higher average accuracy than Ho-CA $(p<0.01)$. Ho-VC shows higher average accuracy than Ho-CA $(p<0.05)$.

Figure 4 shows the results of the analyses of subjects whose average accuracy levels are greater than $0.6,0.7$ and 0.8 in terms of accuracy according to the cue combination

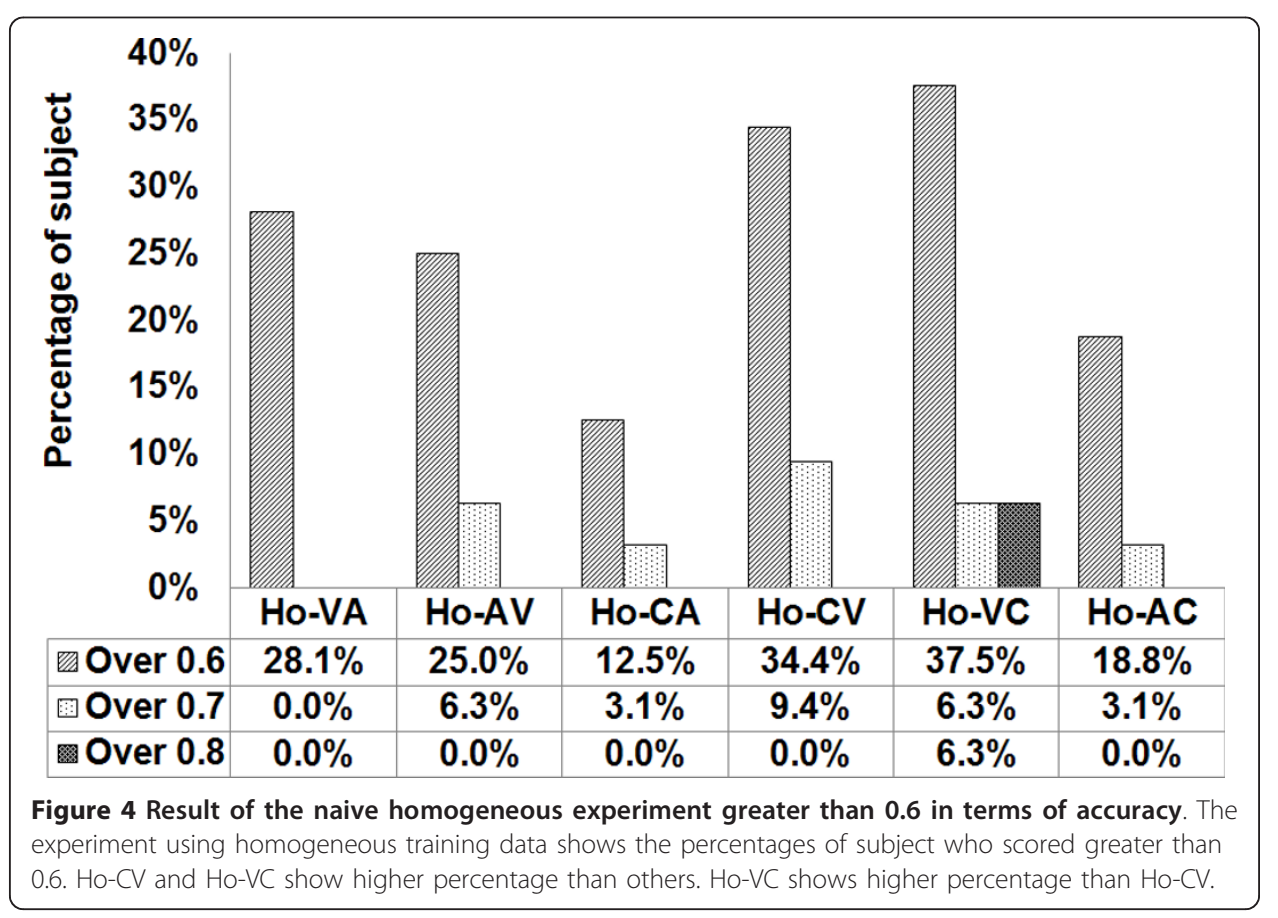


of the Ho-CV combination of a combined cue for training and a visual cue for testing, and the Ho- $\mathrm{VC}$ combination of a visual cue for training and a combined cue for testing. Ho-CV and Ho-VC show higher percentage than others. Ho-VC shows higher percentage than Ho-CV.

The analysis criteria are based on the six combinations described in Table 1. The results shown in Figure 3 are more general considering that they only show the general tendency in terms of the average. In comparison, Figure 4 is more indicative of the actual performance capacity, as the experiment was limited to subjects who scored above a certain level of accuracy.

\section{Naive heterogeneous experiment}

Two of these are specified with different cueing speeds, from which the term heterogeneity applies. Figure 5 shows the accuracy results of the six combinations of audiovisual cues described in Table 1. He-CV shows higher accuracy than He-CA $(p<0.01)$, and $\mathrm{He}-\mathrm{CV}$ shows higher accuracy than He-AC $(p<0.05)$.

Figure 6 shows the result of the analysis of the subjects whose average accuracy is greater than $0.6,0.7$, and 0.8 in terms of accuracy according to the heterogeneous cue combination of the He-CV combination of a combined cue for training and a visual cue for testing, and the $\mathrm{He}-\mathrm{VC}$ combination of a visual cue for training and a combined cue for testing. He-CV and $\mathrm{He}-\mathrm{VC}$ show higher percentage than others. He-CV shows higher percentage than $\mathrm{He}-\mathrm{VC}$.

The analysis criteria are based on the six combinations described in Table 1. The results shown in Figure 5 are more general with respect to the fact that they only show the general tendency in terms of the average. In comparison, Figure 6 is more indicative of the actual performance capacity, as this experiment included only subjects who scored above a certain level of accuracy.

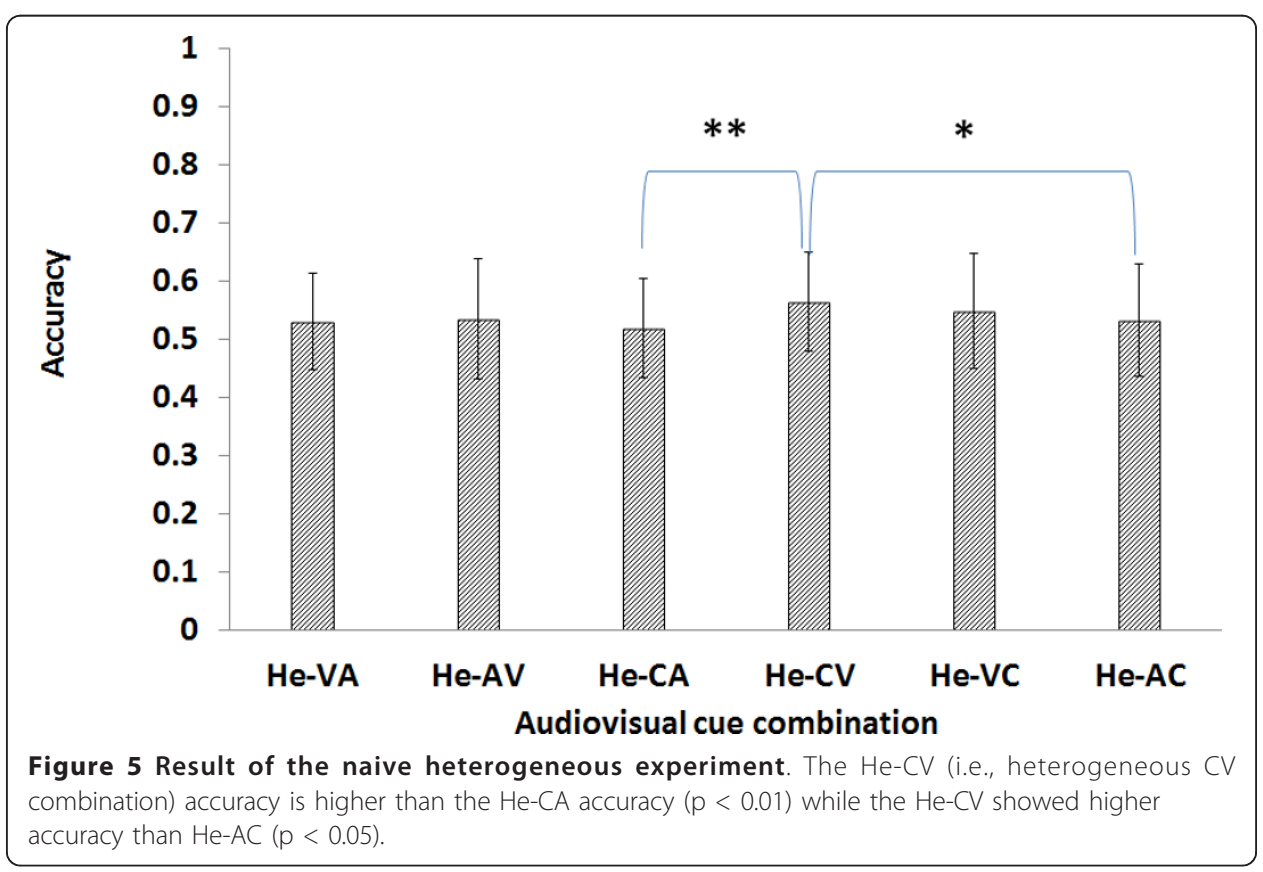




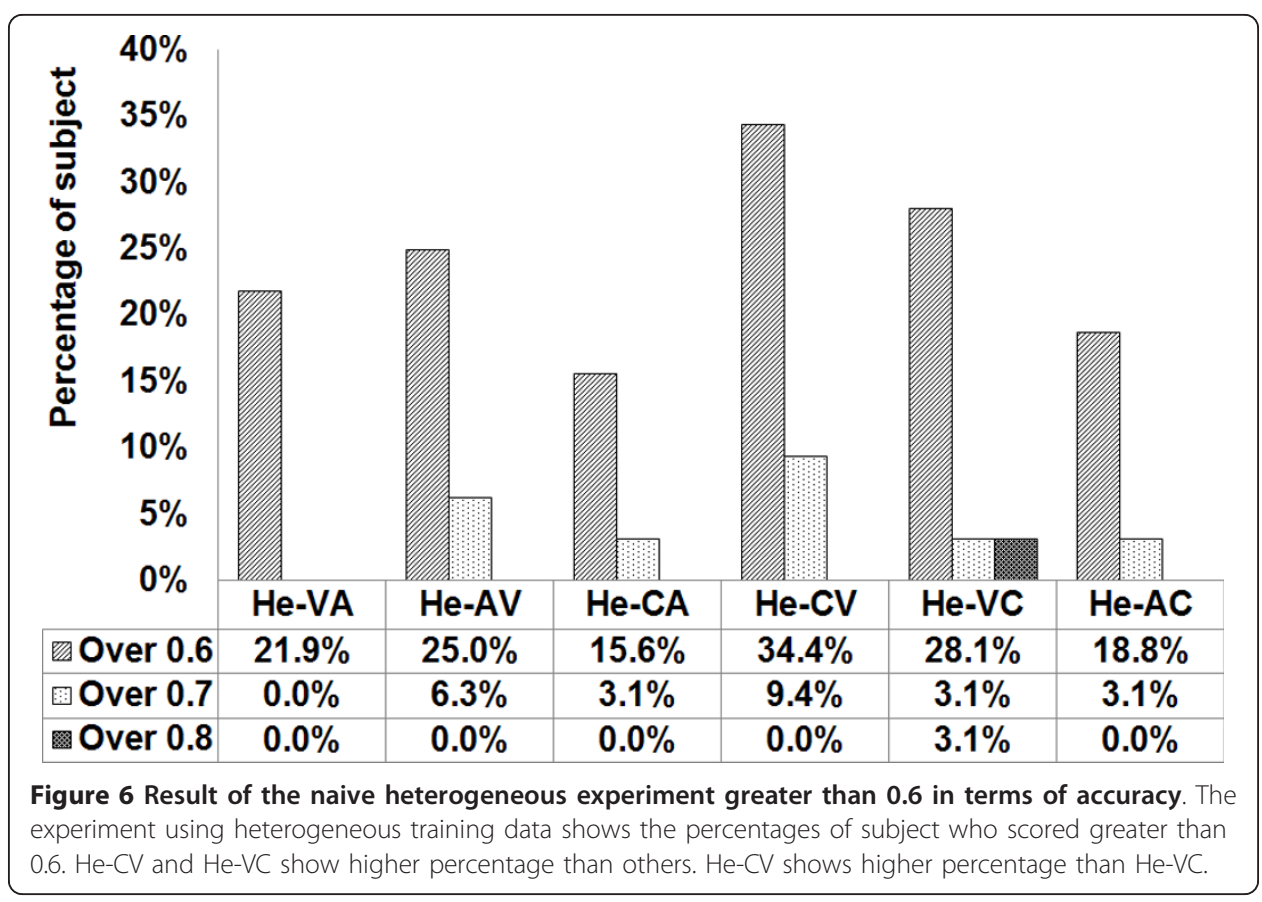

\section{Discussion}

\section{Experienced homogeneous data}

The experienced homogeneous data is cross-compared against 3 classifiers, LS, SVM, and LDA. For the 10 subjects, the CV combination (training on a combined cue with testing was on a visual cue) showed the highest average accuracy. The same types of results were observed in all three of the classifiers (LS, SVM, and LDA). Furthermore, the combinations CA and CV out of the total of six showed the highest level of statistical significance $(p<0.05)$. On the basis of this result, an experiment was carried out on the 32 naive subjects. In this experiment, the comparison was made between experienced subjects using a combined cue as homogeneous training data and naive subjects using a combined cue as homogeneous training data.

\section{Naive homogeneous data}

Out of a total of six combinations in the naive homogeneous training data, the $\mathrm{CV}$ combination showed the highest average accuracy, at 0.562 . The second highest was the VC combinations, at 0.56 . However, when the comparison is confined to the subjects whose average accuracy is greater than 0.6 , the percentage of the $\mathrm{VC}$ combination is higher than that of $\mathrm{CV}$, at $37.5 \%$ and $34.4 \%$, respectively. It is particularly of note that the VC combination included two subjects whose average accuracy score was greater than 0.8 . This shows that the average accuracy alone does not sufficiently explain the individual characteristics of the subjects with all six combinations. The CV combination used a combined cue for the training data and a visual cue for the test data, while the $\mathrm{VC}$ used a visual cue for the training data and a combined cue for the test data. In other words, the combination of $\mathrm{CV}$ and $\mathrm{VC}$ is a cross-combination of combined and visual cues. The results suggest that a combination of these two types of cues results in higher average accuracy. 


\section{Naive heterogeneous data}

The six cue-combinations used in the heterogeneous naive training data experiment showed that the CV combination led to the highest level of accuracy, at 0.564. The second highest level resulted from the VC cue, at 0.549. For the subjects with accuracy scores of 0.6 or above, the CV combination accounted for the highest percentage, at $34.4 \%$ and the second highest was VC at $28.1 \%$. This shows a different trend from the homogeneous training data experiment.

\section{Cross-comparison between the homogenous and heterogeneous experiment}

A cross-comparison between the homogeneous and heterogeneous experiments is carried out in this section to identify the factors that cause the aforementioned differences. Figure 7 shows comparisons of the average accuracy levels between the homogeneous and heterogeneous experiments on the basis of the six combinations described in Table 2.

On average, $\mathrm{CV}$ had the highest scores for both the homogeneous and heterogeneous criteria, and the heterogeneous data showed a higher average level of accuracy. In the case of VC, a contradictory result showed that the homogeneous data showed higher average accuracy levels than the heterogeneous data.

CV was trained on an audio-visual cue and tested on a visual cue. VC was trained on a visual cue and tested on an audio-visual cue. In both cases, there are common conditions that are designed to take advantage of an audio-visual cue and a visual cue as training or test data. In the end, the relationship between the two stimuli had a direct impact on the average performance. In the CV case, there was an improvement in the average performance in the experiment using the heterogeneous training data $(p<$ 0.05), whereas in the VC case, there was decline in the average performance during the experiment using the heterogeneous training data $(p<0.05)$.

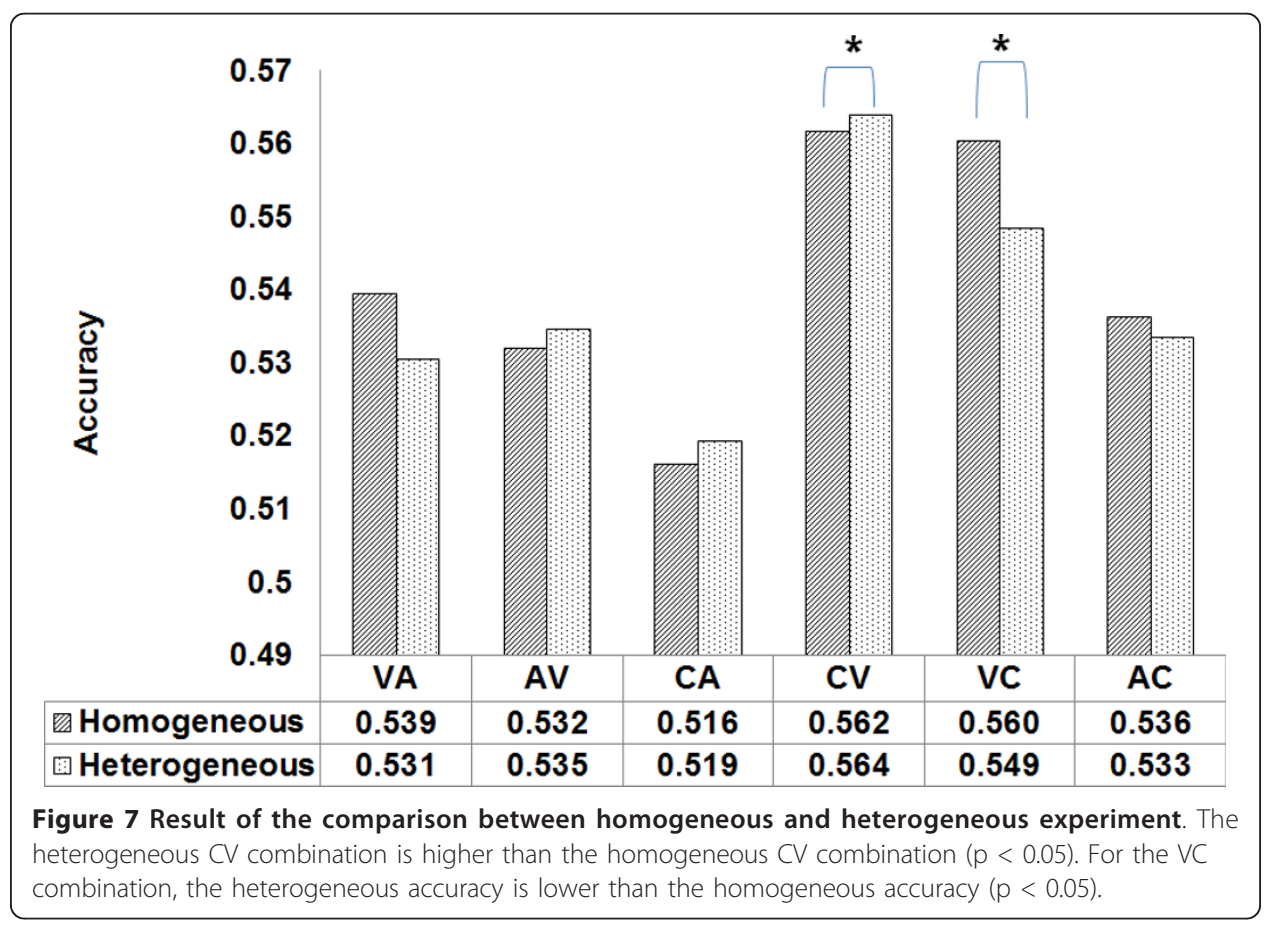


Figure 8 shows the comparisons between homogeneous and heterogeneous training data of the six cue-combinations, including the subjects whose average accuracy score is above 0.6. The CV case, whose training was on an audio-visual cue and whose testing was on a visual cue, showed no difference between the heterogeneous and homogeneous results. This suggests that the effect of the heterogeneity resulting from the cueing speed is minimal. On the other hand, the VC case showed a relatively significant effect in this regard.

The VC case, whose training was on a visual cue and whose performance was evaluated on an audio-visual cue, showed the highest percentage of subjects whose average accuracy score was above 0.6 in the homogeneous training experiment. However, this case showed a lower percentage than the CV case in the heterogeneous training experiment. This suggests that the $\mathrm{VC}$ case, whose training was on visual cue and whose testing was on an audio-visual cue, is not affected by changes in the cueing conditions. This is indicative of more consistent performance.

\section{Conclusions}

When EEG-BCI-based motor imagery training tasks are carried out using naive subjects, the general applicability, stability and consistency of the accuracy levels are regarded the most essential. The set of experiments conducted here concluded that consistent accuracy can be achieved when the training data relies on a heterogeneous combined cue. Randomness of presenting time of the heterogeneous cue raises the power of concentration of the subjects, and this is thought to be the main cause of the consistency in performance.

An accuracy of the difference was $0.002(p<0.05)$ between 0.562 in the homogeneous one and 0.564 in heterogeneous one. A consistent result was obtained when the training data used a combined cue and the test data used a visual cue. Moreover, the

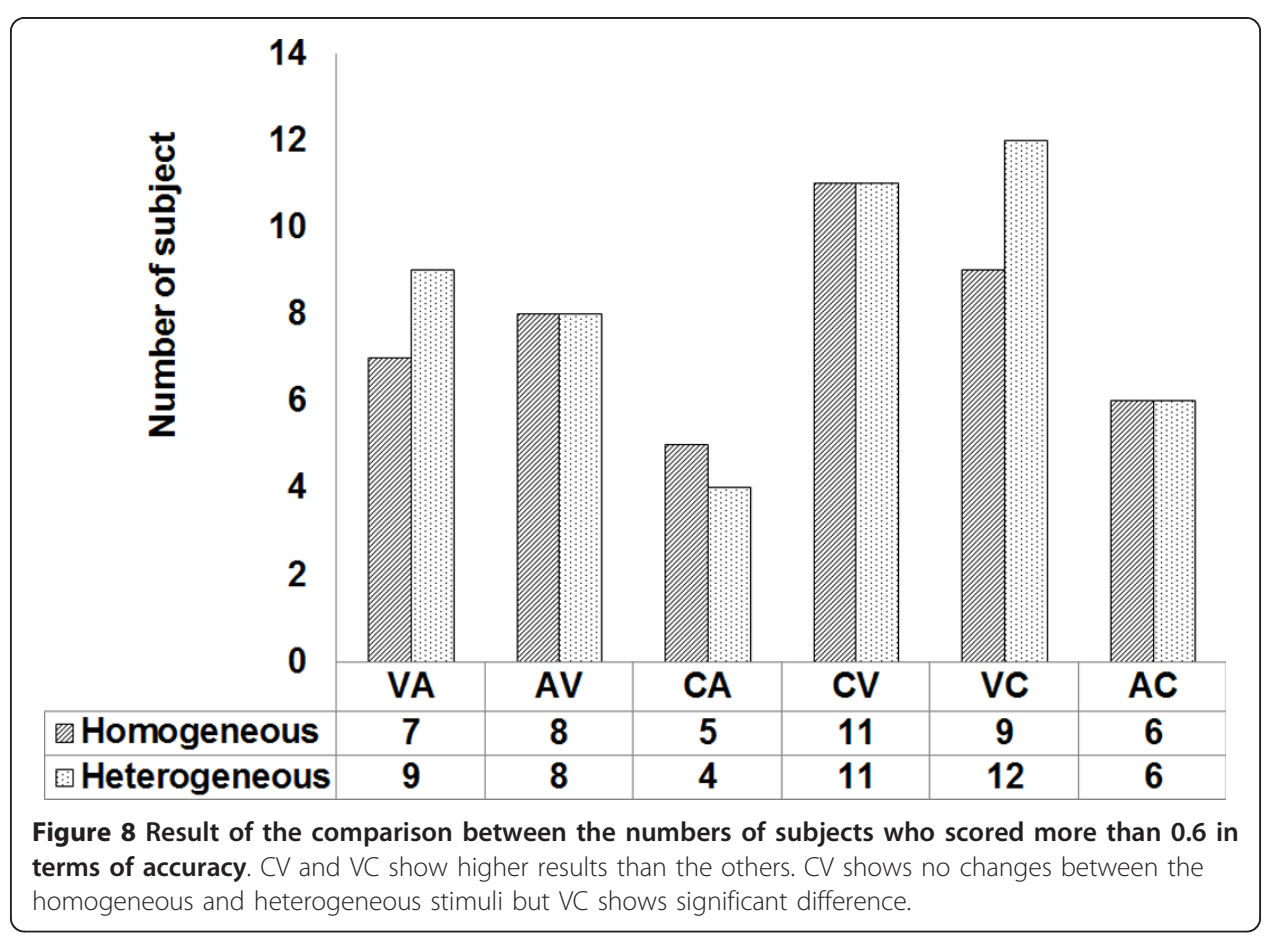


combinations of a combined cue and a visual cue showed the highest at $0.562,0.564$ and the second highest accuracy at 0.56, 0.549.

We propose the use of this measuring methodology of a heterogeneous combined cue for training data and a visual cue as a testing cue by the typical EEG-BCI algorithm on the EEG-BCI system to achieve effectiveness in terms of consistency, stability, cost, time, and resources management without the need for a trial and error process.

\section{Acknowledgements}

None

\section{Authors' contributions}

DC carried out the algorithm implementation and evaluation and drafted the manuscript. YR and YL conceived of the study and participated in its coordination. Both authors participated in the study design and in the data acquisition processes and both read and approved the final manuscript.

\section{Competing interests}

The authors declare that they have no competing interests.

Received: 28 June 2011 Accepted: 12 October 2011 Published: 12 October 2011

\section{References}

1. Dornhege G: Toward brain-computer interfacing U.S.A: The MIT Press; 2007.

2. McFarland DJ, Krusienski DJ, Sarnacki WA, Wolpaw JR: Emulation of computer mouse control with a noninvasive brain-computer interface. Journal of neural engineering 2008, 5:101.

3. Van Gerven M, Farquhar J, Schaefer R, Vlek R, Geuze J, Nijholt A, Ramsey N, Haselager P, Vuurpijl L, Gielen S: The braincomputer interface cycle. J Neural Eng 2009, 6:041001-041001.

4. Pfurtscheller G, Allison BZ, Brunner C, Bauernfeind G, Solis-Escalante T, Scherer R, Zander TO, Mueller-Putz G, Neuper C, Birbaumer N: The hybrid BCl. Frontiers in neuroscience 2010, 4.

5. Ang KK, Guan C, Chua KSG, Ang BT, Kuah C, Wang C, Phua KS, Chin ZY, Zhang H: Clinical study of neurorehabilitation in stroke using EEG-based motor imagery brain-computer interface with robotic feedback. 2010 Annual International Conference of the IEEE Buenos Aires, Argentina. IEEE 2010, 5549-5552.

6. Blankertz B, Losch F, Krauledat M, Dornhege G, Curio G, Muller KR: The Berlin Brain-Computer Interface: accurate performance from first-session in BCI-naive subjects. Biomedical Engineering, IEEE Transactions on 2008, 55(10):2452-2462.

7. Wolpaw JR, MCFarland DJ: Control of a two-dimensional movement signal by a noninvasive brain-computer interface in humans. PNAS-Proceedings of the National Academy of Sciences of the United States of America 2004, 101(51):17849.

8. McFarland DJ, Anderson CW, Muller KR, Schlogl A, Krusienski DJ: BCI meeting 2005-workshop on BCl signal processing: feature extraction and translation. Neural Systems and Rehabilitation Engineering, IEEE Transactions on 2006, 14(2):135-138.

9. Pfurtscheller G, Neuper C: Motor imagery and direct brain-computer communication. Proceedings of the IEEE 2001, 89(7):1123-1134

10. Arroyo S, Lesser RP, Gordon B, Uematsu S, Jackson D, Webber R: Functional significance of the mu rhythm of human cortex: an electrophysiologic study with subdural electrodes. Electroencephalography and clinical Neurophysiology 1993, 87(3):76-87.

11. Muthukumaraswamy SD, Johnson BW, MCNair NA: Mu rhythm modulation during observation of an object-directed grasp. Cognitive Brain Research 2004, 19(2):195-201.

12. Pfurtscheller G, Brunner C, Schlogl A, Lopes da Silva F: Mu rhythm (de) synchronization and EEG single-trial classification of different motor imagery tasks. Neurolmage 2006, 31(1):153-159.

13. Pfurtscheller G, Neuper C: Motor imagery activates primary sensorimotor area in humans. Neuroscience letters 1997, 239(2-3):65-68

14. Ince NF, Tewfik AH, Arica S: Extraction subject-specific motor imagery time-frequency patterns for single trial EEG classification. Computers in biology and medicine 2007, 37(4):499-508.

15. Blankertz B, Tomioka R, Lemm S, Kawanabe M, Muller KR: Optimizing spatial filters for robust EEG single-trial analysis. Signal Processing Magazine, IEEE 2008, 25(1):41-56.

16. Kim J, Kang D, Lee $Y$, Jung H, Lee I, Park H, Kim E, Lee M: Performance Evaluation of EEG-BCI Interface Algorithm in $\mathrm{BCI}$ (Brain Computer Interface)-Naive Subjects. Journal of biomedical engineering research: the official journal of the Korean Society of Medical \& Biological Engineering 2009, 30(5):428-437

17. Lee Y: Performance Evaluation of Motor Imagery Characteristics based EEG-Brain Computer Interface for Ubiquitous Healthcare Service. Ph.D Yonsei, Biomedical Electronics \& Information Lab 2010.

doi:10.1186/1475-925X-10-91

Cite this article as: Choi et al:: Performance evaluation of a motor-imagery-based EEG-Brain computer interface using a combined cue with heterogeneous training data in BCl-Naive subjects. BioMedical Engineering OnLine 2011 10:91. 